\title{
A QUANTUM THEORY OF GRAVITY BASED ON A COMPOSITE MODEL OF LEPTONS AND QUARKS
}

\author{
B. A. ROBSON \\ Department of Theoretical Physics, \\ Research School of Physics and Engineering, \\ The Australian National University, Canberra ACT 0200 \\ brian.robson@anu.edu.au
}

Accepted 15 September 2010

\begin{abstract}
A quantum theory of gravity, based upon a composite model of leptons and quarks, is presented. The model treats leptons and quarks as composites of three kinds of spin- $\frac{1}{2}$ particles (rishons) and/or their antiparticles. A strong color force, mediated by massless hypergluons, binds rishons and/or antirishons together to form colorless leptons or colored quarks, the fundamental particles of the Standard Model. The new model provides a qualitative understanding of the mass hierarchy of the three generations of leptons and quarks. An earlier conjecture that the residual interaction of the strong color force acting between any two colorless particles be identified with the corresponding gravitational interaction, is shown to lead approximately to Newton's law of gravitation.
\end{abstract}

Keywords: Generation model; leptons; quarks; particle generations; composite models; rishons; mass; gravity.

PACS Number(s): 04.50.Kd, 12.38.-t, 12.60.-i, 12.60.Rc

\section{Introduction}

In the standard model ${ }^{1}(\mathrm{SM})$ of particle physics, as emphasized by Wilczek, ${ }^{2}$ hadron masses arise mainly from the energy content of their constituent quarks and/or antiquarks and gluons, in agreement with Einstein's conclusion ${ }^{3}$ : the mass of a body $m$ is a measure of its energy content $E$ and is given by $m=E / c^{2}$. On the other hand, the masses of the fundamental particles of the SM, the leptons, the quarks and the $W$ and $Z$ bosons, are interpreted ${ }^{4}$ in a completely different way, arising from the existence of a so-called Higgs field. ${ }^{5,6}$ This field was introduced to spontaneously break the $\mathrm{U}(1) \times \mathrm{SU}(2)$ local gauge symmetry of the electroweak interaction in the SM to generate the masses of the $W$ and $Z$ gauge bosons. It was also able to cure the associated fermion mass problem: providing finite masses for the leptons and quarks without violating the assumed local gauge invariance.

In a recent paper ${ }^{7}$ it has been suggested that the development of an alternative model, the generation model ${ }^{8,9}$ (GM), which in turn led to a composite generation model $^{10}(\mathrm{CGM})$ of all the fundamental particles of the SM, provides a new paradigm 
for the origin of all mass: the mass of a body arises from the energy content of its constituents, in agreement with Einstein's conclusion, so that there is no need for the existence of a Higgs-type field.

The GM differs from the SM in that it treats quark mixing differently from the method introduced by Cabibbo ${ }^{11}$ and employed in the SM. Essentially, in the GM, the quark mixing is placed in the quark states (wavefunctions) rather than in the weak interactions. This allows a unified and simpler classification of both the leptons and quarks in terms of only three additive quantum numbers, each of which is conserved in all interactions.

The GM predicts that hadrons are composed of mixed-quark states, with the mixing parameters being determined by the Cabibbo-Kobayashi-Maskawa matrix elements, as used in the SM. In this way the universality of the weak interaction is maintained and the predicted transition probabilities for weak interaction processes are the same in the GM as those of the SM. The notion of mixed-quark states in hadrons provides an understanding of why the neutron is heavier than the proton, contrary to expectation arising from quark mass systematics. The GM also predicts that the strange quark content of the proton is about $1.7 \%$, which is significantly larger than expected from the SM. ${ }^{12}$

In addition, the unified classification scheme of the GM led to the development of a CGM of leptons and quarks. This is not possible in terms of the non-unified classification scheme of the SM, involving different additive quantum numbers for leptons than for quarks and the non-conservation of some additive quantum numbers, such as strangeness, in the case of quarks.

In the CGM the fundamental particles of the SM have a substructure, consisting of massless rishons and/or antirishons bound together by strong color interactions, mediated by massless hypergluons. These composite particles, the leptons, quarks and the $W$ and $Z$ vector bosons, are assumed to be colorless. This model is analogous to the SM in which quarks and/or antiquarks are bound together by strong color interactions, mediated by massless gluons, to form hadrons. Since the mass of a hadron arises mainly from the energy of its constituent quarks and/or antiquarks and gluons, the CGM suggests that the mass $m$ of a lepton, quark or vector boson arises similarly from a characteristic energy $E$ associated with its constituent rishons and/or antirishons and hypergluons, given by $m=E / c^{2}$.

The above new paradigm for the origin of all mass suggests that the quantum mechanical nonlocalization of the constituent rishons of a fundamental particle of the SM gives rise to both its mass and also to a residual interaction between any two such particles.

The mass of each fundamental particle of the SM is related to the incomplete cancellation of the "intra-fermion" strong color interactions acting between its constituent rishons and/or antirishons, and is expected to be greater if the degree of localization of the constituent rishons and/or antirishons is less (i.e. the rishons and/or antirishons are on the average more widely separated). This is a consequence of the nature of the strong interactions, which are assumed to possess the property 
of "asymptotic freedom". ${ }^{13,14}$ Furthermore, the large variation in the masses of the fundamental particles of the SM indicates that the mass of a particle is extremely sensitive to the degree of localization of its constituents.

The proposed residual interaction, arising from the incomplete cancellation of the "inter-fermion" color interactions acting between the rishons and/or antirishons of one particle and those of another particle, has been identified ${ }^{7}$ with the usual gravitational interaction, having several properties associated with that interaction: universality, infinite range and very weak strength.

Within the framework of the CGM there exists considerable indirect evidence for the expected relative masses of the fundamental fermions of the SM.

Firstly, the electron consists of three antirishons, each carrying identical electric charge in addition to their color charges, while the electron neutrino consists of three electrically neutral antirishons. It is predicted that the electromagnetic interaction in the electron case will cause the rishons to be less localized than those constituting the electron neutrino so that the electron will have a substantially greater characteristic energy and correspondingly a greater mass than the electron neutrino. Similarly, the muon and the tau lepton are expected to have considerably greater masses than their corresponding neutrinos.

Secondly, the up, charmed and top quarks, each containing two identical electrically charged rishons, are expected to have a greater mass than their weak isospin partners, the down, strange and bottom quark respectively, which contain only one electrically charged antirishon. This is true, ${ }^{9}$ provided one takes into account quark mixing in the case of the up and down quarks.

On the other hand, the larger masses of the quarks (with only one or two identical electrically charged rishons) relative to the charged leptons (with three identical electrically charged rishons) of the same generation are difficult to understand within the framework of the CGM. This discrepancy indicates that the leptons and quarks have different color-charge structures and that the CGM requires modification.

In Sec. 2 we shall present a more consistent composite model of leptons and quarks (CGM-2), which provides an alternative structure for quarks that removes the above mass discrepancy. This new model provides a qualitative understanding of the mass hierarchy of the three generations of leptons and quarks.

In the CGM-2, it is assumed that instead of consisting of a triad of rishons having different color charges, with the higher generations having an additional colorless rishon-antirishon pair as in the CGM, the quarks consist of one rishon, carrying a color charge, and one or more colorless rishon-antirishon pairs.

In addition, we adopt a different color structure for the rishons from that assumed in the CGM: all three rishons, $T, V$ and $U$ now carry a color charge, red, green or blue, while their antiparticles carry an anticolor charge, antired, antigreen or antiblue. This requires the composite quarks to have a color charge so that the dominant residual interaction between quarks is essentially the same as that be- 
tween rishons, and the composite quarks behave more nearly like the fundamental quarks of the SM. In particular, the colored quarks form colorless hadrons, basically as in the SM, and this overcomes the difficulties associated with describing the confining properties of the colorless quarks assumed in the CGM.

In the CGM-2, we retain the term "hypergluon" as the mediator of the color interaction, rather than the term "gluon" employed in the SM, because it is the rishons rather than the quarks which carry an elementary color charge.

Section 3 discusses the nature of the residual interaction associated with the usual gravitational interaction with respect to the different quark color structures assumed in the CGM-2. It will be argued that the CGM-2 leads approximately to Newton's law of gravitation. Finally, Sec. 4 states the conclusions.

\section{Composite Generation Model-2 (CGM-2)}

The CGM-2 treats leptons and quarks as composites of the same three kinds of spin$\frac{1}{2}$ rishons and/or antirishons as the CGM and Table 1 gives the additive quantum numbers allotted to these rishons. These quantum numbers are the same as in the CGM. It should be noted that for a given rishon having additive quantum number $N$, the corresponding antirishon has the additive quantum number $-N$. These additive quantum numbers satisfy the two electric charge $(Q)$ equations:

$$
Q=I_{3}+\frac{1}{2}(p+g)=i_{3}+\frac{1}{2} p,
$$

where $I_{3}$ and $i_{3}$ are the third components of strong isospin $(I)$ and weak isospin $(i)$, respectively, and $p$ and $g$ are the particle and generation quantum numbers of the GM.

We shall now indicate how mass considerations lead to different possible rishon and/or antirishon structures for the three generations of leptons and quarks. In the CGM-2, contrary to the CGM, antirishons are assumed to carry only anticolors not colors. This leads to different color structures for many of the fundamental particles of the SM, especially the quarks which now carry a color charge.

\subsection{First generation}

The first generation of the fundamental particles of the SM consists of two leptons, the electron $\left(e^{-}\right)$and the electron neutrino $\left(\nu_{e}\right)$, and two quarks, the up quark $(u)$

Table 1. CGM-2 additive quantum numbers for rishons.

\begin{tabular}{crrrrr}
\hline Rishon & $Q$ & $I_{3}$ & $p$ & $g$ & $i_{3}$ \\
\hline$T$ & $+\frac{1}{3}$ & $+\frac{1}{6}$ & $+\frac{1}{3}$ & 0 & $+\frac{1}{6}$ \\
$V$ & 0 & $-\frac{1}{6}$ & $+\frac{1}{3}$ & 0 & $-\frac{1}{6}$ \\
$U$ & 0 & $+\frac{1}{3}$ & $+\frac{1}{3}$ & -1 & $-\frac{1}{6}$ \\
\hline
\end{tabular}


Table 2. CGM-2 of first generation of leptons and quarks.

\begin{tabular}{ccccccc}
\hline Particle & Structure & $Q$ & $I_{3}$ & $p$ & $g$ & $i_{3}$ \\
\hline$e^{+}$ & $T T T$ & +1 & $+\frac{1}{2}$ & +1 & 0 & $+\frac{1}{2}$ \\
$u$ & $T T \bar{T}$ & $+\frac{2}{3}$ & $+\frac{1}{2}$ & $+\frac{1}{3}$ & 0 & $+\frac{1}{2}$ \\
$\bar{d}$ & $T \bar{V} \bar{V}$ & $+\frac{1}{3}$ & $+\frac{1}{2}$ & $-\frac{1}{3}$ & 0 & $+\frac{1}{2}$ \\
$\nu_{e}$ & $\bar{V} \bar{V} \bar{V}$ & 0 & $+\frac{1}{2}$ & -1 & 0 & $+\frac{1}{2}$ \\
$\overline{\nu_{e}}$ & $V V V$ & 0 & $-\frac{1}{2}$ & +1 & 0 & $-\frac{1}{2}$ \\
$d$ & $\bar{T} V V$ & $-\frac{1}{3}$ & $-\frac{1}{2}$ & $+\frac{1}{3}$ & 0 & $-\frac{1}{2}$ \\
$\bar{u}$ & $\bar{T} \bar{T} V$ & $-\frac{2}{3}$ & $-\frac{1}{2}$ & $-\frac{1}{3}$ & 0 & $-\frac{1}{2}$ \\
$e^{-}$ & $\bar{T} \bar{T} \bar{T}$ & -1 & $-\frac{1}{2}$ & -1 & 0 & $-\frac{1}{2}$ \\
\hline
\end{tabular}

and the down quark $(d)$. The two leptons and the two quarks are both weak isospin partners, i.e. have $i_{3}= \pm \frac{1}{2}$.

In the CGM-2 the same rishon structure is assumed for both the leptons and the quarks of the first generation as in the CGM. These structures are given in Table 2. Here the structures contain two kinds of spin- $\frac{1}{2}$ rishons: (i) a $T$-rishon with $Q=+\frac{1}{3}$, (ii) a $V$-rishon with $Q=0$ and their corresponding antiparticles (denoted in the usual way by a bar over the defining particle symbol) $\bar{T}$ and $\bar{V}$.

In the CGM-2 as in the CGM, each lepton of the first generation is assumed to be colorless, consisting of three rishons (or antirishons), each with a different color (or anticolor), analogous to the baryons (or antibaryons) of the SM. These leptons are built out of $T$ - and $V$-rishons or their antiparticles $\bar{T}$ and $\bar{V}$, all of which have generation quantum number $g=0$.

It is envisaged that each lepton of the first generation, as in the CGM, exists in an antisymmetric three-particle color state, which physically assumes a quantum mechanical triangular distribution of the three differently colored identical rishons (or antirishons), since each of the three color interactions between pairs of rishons (or antirishons) is expected to be strongly attractive. ${ }^{15}$ As indicated earlier, the charged leptons are predicted to have larger masses than the uncharged leptons, since the electromagnetic interaction in the charged leptons will cause their constituent rishons (or antirishons) to be less localized than those constituting the uncharged leptons, leading to a substantially greater characteristic energy and a correspondingly greater mass.

On the other hand, in the CGM-2, each quark of the first generation is assumed to have a different color charge structure to that assumed in the CGM. In particular, the $\bar{V}$-antirishon is assumed to carry an anticolor charge, rather than a color charge, as in the CGM. Thus it is envisaged that each quark is a composite of a colored rishon and a colorless rishon-antirishon pair, $(\bar{V} T)$ or $(\bar{T} V)$, so that in the CGM-2, the quarks carry a color charge. This means that the dominant residual interaction between these quarks is essentially the same as that between rishons and consequently these composite quarks behave more nearly like the fundamental quarks of the SM. 
In order to preserve the universality of the weak interaction processes involving the first generation of quarks, e.g. the transition $u \rightarrow d+W^{+}$, it is assumed that the first generation quarks have the general forms:

up quark: $T_{c}\left(T_{c^{\prime}} \bar{V}_{\bar{c}^{\prime}}\right)$, down quark: $V_{c}\left(V_{c^{\prime}} \bar{T}_{\bar{c}^{\prime}}\right)$, with $\quad c^{\prime} \neq c$.

Thus a red $u$-quark and a red $d$-quark have the general color structures:

$$
u_{r}=T_{r}\left(T_{g} \bar{V}_{\bar{g}}+T_{b} \bar{V}_{\bar{b}}\right) / \sqrt{2},
$$

and

$$
d_{r}=V_{r}\left(V_{g} \bar{T}_{\bar{g}}+V_{b} \bar{T}_{\bar{b}}\right) / \sqrt{2},
$$

respectively. For $u_{r} \rightarrow d_{r}+W^{+}$, one has the two transitions:

$$
T_{r} T_{g} \bar{V}_{\bar{g}} \rightarrow V_{r} V_{b} \bar{T}_{\bar{b}}+T_{r} T_{g} T_{b} \bar{V}_{\bar{r}} \bar{V}_{\bar{g}} \bar{V}_{\bar{b}}
$$

and

$$
T_{r} T_{b} \bar{V}_{\bar{b}} \rightarrow V_{r} V_{g} \bar{T}_{\bar{g}}+T_{r} T_{g} T_{b} \bar{V}_{\bar{r}} \bar{V}_{\bar{g}} \bar{V}_{\bar{b}}
$$

which take place with equal probabilities. In these transitions, the $W^{+}$boson is assumed to have a similar structure to that assumed in the CGM, consisting of a colorless set of three $T$-rishons and a colorless set of three $\bar{V}$-antirishons. The transitions take place by the simultaneous production of three colorless rishonantirishon pairs as in the CGM.

The new color charge structure of the quarks is expected to lead to a quantum mechanical linear distribution of the constituent rishons and/or antirishons, corresponding to a considerably larger mass than that of the leptons, since the constituents of the quarks are now less localized. This is a consequence of the expected character (i.e. attractive/repulsive) of the color interactions at small distances. ${ }^{15}$ The general rules for small distances of separation are:

(i) rishons (or antirishons) of like colors (or anticolors) repel: those having different colors (or anticolors) attract, unless their colors (or anticolors) are interchanged and the two rishons (or antirishons) do not exist in an antisymmetric color state (e.g. as in the case of leptons);

(ii) rishons and antirishons of opposite colors attract but otherwise repel.

Furthermore, the electromagnetic interaction occurring within the up quark, leads one to expect it to have a larger mass than that of the down quark.

\subsection{Second generation}

The second generation of the fundamental particles of the SM consists of two leptons, the muon $\left(\mu^{-}\right)$and the muon neutrino $\left(\nu_{\mu}\right)$, and two quarks, the charmed quark $(c)$ and the strange quark $(s)$. The two leptons and the two quarks are also weak isospin partners. 
Table 3. CGM-2 of second generation of leptons and quarks.

\begin{tabular}{cccccc}
\hline Particle & Structure & $Q$ & $p$ & $g$ & $i_{3}$ \\
\hline$\mu^{+}$ & $T T T \Pi$ & +1 & +1 & \pm 1 & $+\frac{1}{2}$ \\
$c$ & $T T \bar{V} \Pi$ & $+\frac{2}{3}$ & $+\frac{1}{3}$ & \pm 1 & $+\frac{1}{2}$ \\
$\bar{s}$ & $T \bar{V} \bar{V} \Pi$ & $+\frac{1}{3}$ & $-\frac{1}{3}$ & \pm 1 & $+\frac{1}{2}$ \\
$\nu_{\mu}$ & $\bar{V} \bar{V} \bar{V} \Pi$ & 0 & -1 & \pm 1 & $+\frac{1}{2}$ \\
$\bar{\nu}_{\mu}$ & $V V V \Pi$ & 0 & +1 & \pm 1 & $-\frac{1}{2}$ \\
$s$ & $\bar{T} V V \Pi$ & $-\frac{1}{3}$ & $+\frac{1}{3}$ & \pm 1 & $-\frac{1}{2}$ \\
$\bar{c}$ & $\bar{T} \bar{T} V \Pi$ & $-\frac{2}{3}$ & $-\frac{1}{3}$ & \pm 1 & $-\frac{1}{2}$ \\
$\mu^{-}$ & $\bar{T} \bar{T} \bar{T} \Pi$ & -1 & -1 & \pm 1 & $-\frac{1}{2}$ \\
\hline
\end{tabular}

In the CGM-2, a different rishon structure is assumed for both the leptons and the quarks of the second generation from that proposed in the CGM. In the CGM, the rishon structure of the third generation is very similar to that of the second generation, so that the masses of the second and third generation particles are expected to be very similar. In order to overcome this mass discrepancy with observation, it is proposed that the rishon structure of the leptons and quarks of the second generation should be a quantum mechanical equal mixture of both the rishon structures previously assumed for each of the second and third generation particles in the CGM. These structures are given in Table 3.

Thus, for example, the rishon structure of the $\mu^{+}$particle is now TTT $\Pi$, where

$$
\Pi=[(\bar{U} V)+(\bar{V} U)] / \sqrt{2}
$$

and each particle of the second generation is a similar quantum mechanical mixture of $g= \pm 1$ components.

The rishon structures of the second generation particles are the same as the corresponding particles of the first generation plus the addition of a colorless rishonantirishon pair, $\Pi$, which is a quantum mechanical mixture of $(\bar{U} V)$ and $(\bar{V} U)$, which have $p=Q=0$ but $g= \pm 1$, respectively. In this way, the pattern of the first generation is repeated for the second generation. It should be noted that for any given transition the generation quantum number is required to be conserved, although each particle of the second generation now has two possible values of $g$.

For example, the decay

$$
\mu^{+} \rightarrow \bar{\nu}_{\mu}+W^{+}
$$

at the rishon level may be written as

$$
T T T \Pi \rightarrow V V V \Pi+T T T \bar{V} \bar{V} \bar{V},
$$

which proceeds via the two transitions:

$$
T T T(\bar{U} V) \rightarrow V V V(\bar{U} V)+T T T \bar{V} \bar{V} \bar{V}
$$


and

$$
T T T(\bar{V} U) \rightarrow V V V(\bar{V} U)+T T T \bar{V} \bar{V} \bar{V},
$$

which take place with equal probabilities. In each case, the additional colorless rishon-antirishon pair, $(\bar{U} V)$ or $(\bar{V} U)$, essentially act as spectators during the weak interaction process.

It is envisaged that each lepton of the second generation basically exists in an antisymmetric three-particle color state, which physically assumes quantum mechanically a triangular distribution of the three differently colored identical rishons (or antirishons), as for the corresponding particle of the first generation. The additional colorless rishon-antirishon pair, $(\bar{U} V)$ or $(\bar{V} U)$, is expected to be attached externally to this triangular distribution, leading quantum mechanically to a less localized distribution of the constituent rishons and/or antirishons, so that the particle has a significantly larger mass than its corresponding first generation particle.

Each quark of the second generation has a similar structure to that of the corresponding quark of the first generation, with the additional colorless rishonantirishon pair, $(\bar{U} V)$ or $(\bar{V} U)$, attached quantum mechanically so that the whole rishon structure is essentially a linear distribution of the constituent rishons and antirishons. This structure is expected to be less localized, leading to a larger mass relative to that of the corresponding quark of the first generation, with the charmed quark having a greater mass than the strange quark, arising from the electromagnetic repulsion of its constituent two charged $T$-rishons.

It should be noted that the intrinsic parity of the strange quark is envisaged to be the same as that in the CGM and opposite to that of the down quark, so that the long-lived neutral kaon $\left(K_{L}^{0}\right)$ may decay to two charged pions without $\mathrm{CP}$ violation. ${ }^{16}$

\subsection{Third generation}

The third generation of the fundamental particles of the SM consists of two leptons, the tau particle $\left(\tau^{-}\right)$and the tau neutrino $\left(\nu_{\tau}\right)$, and two quarks, the top quark $(t)$ and the bottom quark $(b)$. These two leptons and two quarks are also weak isospin partners.

In the CGM-2, a different rishon structure is proposed for both the leptons and the quarks of the third generation from that suggested in the CGM. In order to qualitatively describe the significantly larger masses of the third generation particles, it is proposed that these particles have the rishon structures given in Table 4.

Thus, for example, the rishon structure of the $\tau^{+}$particle is

$$
T T T \Pi \Pi=T T T[(\bar{U} V)(\bar{U} V)+(\bar{U} V)(\bar{V} U)+(\bar{V} U)(\bar{U} V)+(\bar{V} U)(\bar{V} U)] / 2
$$

and each particle of the third generation is a similar quantum mechanical mixture of $g=0, \pm 2$ components.

The rishon structures of the third generation particles are the same as the corresponding particles of the first generation plus the addition of two rishonantirishon pairs, which are a quantum mechanical mixture of $(\bar{U} V)$ and $(\bar{V} U)$ and, 
Table 4. CGM-2 of third generation of leptons and quarks.

\begin{tabular}{ccrccc}
\hline Particle & Structure & $Q$ & $p$ & $g$ & $i_{3}$ \\
\hline$\tau^{+}$ & $T T T \Pi \Pi$ & +1 & +1 & $0, \pm 2$ & $+\frac{1}{2}$ \\
$t$ & $T T \bar{V} \Pi \Pi$ & $+\frac{2}{3}$ & $+\frac{1}{3}$ & $0, \pm 2$ & $+\frac{1}{2}$ \\
$\bar{b}$ & $T \bar{V} \bar{V} \Pi \Pi$ & $+\frac{1}{3}$ & $-\frac{1}{3}$ & $0, \pm 2$ & $+\frac{1}{2}$ \\
$\nu_{\tau}$ & $\bar{V} \bar{V} \bar{V} \Pi \Pi$ & 0 & -1 & $0, \pm 2$ & $+\frac{1}{2}$ \\
$\bar{\nu}_{\tau}$ & $V V V \Pi \Pi$ & 0 & +1 & $0, \pm 2$ & $-\frac{1}{2}$ \\
$b$ & $\bar{T} V V \Pi \Pi$ & $-\frac{1}{3}$ & $+\frac{1}{3}$ & $0, \pm 2$ & $-\frac{1}{2}$ \\
$\bar{t}$ & $\bar{T} \bar{T} V \Pi \Pi$ & $-\frac{2}{3}$ & $-\frac{1}{3}$ & $0, \pm 2$ & $-\frac{1}{2}$ \\
$\tau^{-}$ & $\bar{T} \bar{T} \bar{T} \Pi \Pi$ & -1 & -1 & $0, \pm 2$ & $-\frac{1}{2}$ \\
\hline
\end{tabular}

as for the second generation, are assumed to be colorless and have $p=Q=0$ but $g= \pm 1$. In this way, the pattern of the first and second generation is continued for the third generation. Again it should be noted that for any given transition that the generation quantum number is required to be conserved, although each particle of the third generation now has three possible values of $g$. Furthermore, since the conservation of $g$ in weak interactions is a consequence of the conservation ${ }^{10}$ of the three kinds of rishons $(T, V$ and $U)$, transitions between the third generation and the first generation via weak interactions are prohibited even for $g=0$ components of third generation particles.

It is considered that each lepton of the third generation basically exists in an antisymmetric three-particle color state, which physically assumes a quantum mechanical triangular distribution of the three differently colored identical rishons (or antirishons), as for the corresponding particles of the first and second generations. The two additional colorless rishon-antirishon pairs, $(\bar{U} V)(\bar{U} V),(\bar{U} V)(\bar{V} U)$ or $(\bar{V} U)(\bar{V} U)$, are expected to be attached externally to this triangular distribution, leading to a considerably less localized quantum mechanical distribution of the constituent rishons and/or antirishons, so that the particle has a significantly larger mass than its corresponding second generation particle.

Each quark of the third generation has a similar structure to that of the first generation, with the additional two rishon-antirishon pairs $(\bar{U} V)$ and/or $(\bar{V} U)$ attached quantum mechanically so that the whole rishon structure is essentially a linear distribution of the constituent rishons and antirishons. This structure is expected to be even less localized, leading to a larger mass relative to that of the corresponding quark of the second generation, with the top quark having a greater mass than the bottom quark, arising from the electromagnetic repulsion of its constituent two charged $T$-rishons. It should be noted that the intrinsic parity of the bottom quark is now envisaged to be opposite to that in the CGM and the same as that of the down quark.

The color structures of both second and third generation leptons and quarks have been chosen so that the weak interactions are universal. In each case, the 
additional colorless rishon-antirishon pairs, $(\bar{U} V)$ and/or $(\bar{V} U)$, essentially act as spectators during any weak interaction process. In this way, the pattern of the first generation is repeated for the second and third generation.

\section{Gravity}

In an earlier paper ${ }^{7}$ it was proposed that the residual interaction, arising from the incomplete cancellation of the strong color interactions binding the constituents (rishons and/or antirishons) of a colorless particle, could be identified with the usual gravitational interaction, since it had several properties associated with that interaction: universality, infinite range and very weak strength.

The mass of a body of ordinary matter is essentially the total mass of its constituent electrons, protons and neutrons. It should be noted that the mass of an electron, proton or neutron will depend upon the environment in which the particle exists: e.g. the mass of a proton in an atom of helium will differ slightly from that of a proton in an atom of lead. In the CGM-2, each of these three particles is considered to be colorless. The electron is composed of three $\bar{T}$-antirishons, each carrying a different anticolor charge, antired, antigreen or antiblue. Both the proton and neutron are envisaged (as in the SM) to be composed of three quarks, each carrying a different color charge, red, green or blue. All three particles are assumed to be essentially in a three-color antisymmetric state, so that their behavior with respect to the strong color interactions is expected basically to be the same. This similar behavior suggests that the proposed residual interaction has several properties associated with the usual gravitational interaction. Indeed, it is plausible to provide a heuristic derivation of Newton's law of universal gravitation, based upon the above residual interaction.

Firstly, the residual interaction between any two of the above colorless particles, arising from the color interactions acting between the rishons and/or antirishons of one fermion and the rishons and/or antirishons of the other fermion, is predicted to be of a universal character.

Secondly, assuming that the strong color fields are almost completely cancelled at large distances, it seems plausible that the residual interaction, mediated by massless hypergluons, should have an infinite range, and tend to zero as $1 / r^{2}$. These properties may be attributed to the fact that the constituents of each colorless particle are very strongly localized so that the strength of the residual interaction is extremely weak, and consequently the hypergluon self-interactions are also practically negligible. This means that one may consider the color interactions using a perturbation approach and Feynman diagram techniques. It is envisaged that the residual color interaction is the sum of all the two-particle color charge interactions, each of which may be treated perturbatively, i.e. as a single hypergluon exchange. Using the color factors ${ }^{15}$ appropriate for the $\mathrm{SU}(3)$ gauge field, one finds that the residual color interactions between any two colorless particles (electron, neutron or proton) are each attractive. 
Since the mass of a body of ordinary matter is essentially the total mass of its constituent electrons, neutrons and protons, the total interaction between two bodies of masses, $m_{1}$ and $m_{2}$, will be the sum of all the two-particle contributions so that the total interaction will be proportional to the product of these two masses, $m_{1} m_{2}$, provided that each two-particle interaction contribution is also proportional to the product of the masses of the two particles.

This latter requirement may be understood if each electron, neutron or proton is considered physically to be essentially a quantum mechanical triangular distribution of three differently colored rishons or antirishons. In this case, each particle may be viewed as a distribution of three color charges throughout a small volume of space with each color charge having a certain probability of being at a particular point, determined by its corresponding color wavefunction. The total residual interaction between the two colorless particles will then be the sum of all the intrinsic interactions acting between a particular triangular distribution of one particle with that of the other particle.

Now the mass $m$ of each colorless particle is envisaged to be given by $m=E / c^{2}$, where $E$ is a characteristic energy, determined by the degree of localization of its constituent rishons and/or antirishons. Thus the significant volume of space occupied by the triangular distribution of the three differently colored rishons or antirishons is larger the greater the mass of the particle. Moreover, due to antiscreening effects ${ }^{13,14}$ of the strong color fields, the average strength of the color charge within each unit volume of the larger localized volume of space will be increased. If one assumes that the mass of a particle is proportional to the integrated sum of the intra-fermion interactions within the significant volume of space occupied by the triangular distribution, then the total residual interaction between two such colorless particles will be proportional to the product of their masses.

Thus the residual color interaction between two colorless bodies of masses, $m_{1}$ and $m_{2}$, is proportional to the product of these masses and moreover is expected to depend approximately as the inverse square of their distance of separation $r$, i.e. as $1 / r^{2}$, in accordance with Newton's law of gravitation. The approximate dependence on the inverse square law is expected to arise from the effect of hypergluon selfinteractions, especially for large separations. Such deviations from an inverse square law do not occur for electromagnetic interactions, since there are no corresponding photon self-interactions.

\section{Conclusion and Discussion}

A more consistent composite generation model (CGM-2) has been developed, which removes a mass discrepancy found ${ }^{7}$ for an earlier similar model (CGM). ${ }^{10}$ The new model provides a qualitative understanding of the mass hierarchy of the three generations of leptons and quarks. The essential difference between the two models is the different color charges allotted to the rishons and antirishons, which are the basic building blocks of the leptons and quarks. 
In the CGM-2, all three kinds of rishons carry a color charge, red, green or blue, while their antiparticles carry an anticolor charge, antired, antigreen or antiblue. The main consequence of this, is that the composite quarks carry a color charge so that the dominant residual interaction between quarks is essentially the same as that between rishons, and the composite quarks behave more nearly like the fundamental quarks of the SM. In particular, the colored quarks form colorless hadrons (as in the SM), and this overcomes the difficulties associated with describing the confining properties of the colorless quarks assumed in the CGM. The term "hypergluon" as the mediator of the color interaction rather than the term "gluon" as in the SM, is retained in the CGM-2, since it is the rishons rather than the quarks, which carry an elementary color charge. However, the term "super-strong," indicating the relative strength of the color interaction between rishons to the "strong" color interaction between quarks in the SM, has been replaced by "strong," since in the CGM-2, the color interactions between rishons and those between quarks are basically the same.

An earlier conjecture ${ }^{7}$ that the residual interaction of the strong color force acting between any two colorless particles be identified with the corresponding gravitational interaction, has been argued to lead approximately to Newton's law of gravitation. The residual color interaction is expected to be only approximately equivalent to Newton's inverse square law, $1 / r^{2}$, since the effect of hypergluon selfinteractions should modify this law slightly. This kind of deviation from an inverse square law, arising from the occurrence of hypergluon self-interactions, distinguishes the residual color interaction from that of the electromagnetic interaction for which there are no corresponding photon self-interactions. It is envisaged that this deviation from an inverse square law may account for such observations as the Pioneer anomaly $^{17}$ and the galaxy rotation problem. ${ }^{18}$

\section{Acknowledgments}

I am grateful to N. H. Fletcher, D. Robson and L. J. Tassie for helpful discussions.

\section{References}

1. K. Gottfried and V. F. Weisskopf, Concepts of Particle Physics, Vol. 1 (Oxford University Press, New York, 1984).

2. F. Wilczek, Nature 433 (2005) 239.

3. A. Einstein, Ann. Physik 18 (1905) 639.

4. I. J. R. Aitchison and A. J. G. Hey, Gauge Theories in Particle Physics (Adam Hilger Ltd., Bristol, 1982).

5. F. Englert and R. Brout, Phys. Rev. Lett. 13 (1964) 321.

6. P. W. Higgs, Phys. Rev. Lett. 13 (1964) 508.

7. B. A. Robson, Int. J. Mod. Phys. E 18 (2009) 1773.

8. B. A. Robson, Int. J. Mod. Phys. E 11 (2002) 555; B. A. Robson, Int. J. Mod. Phys. E 13 (2004) 999.

9. P. W. Evans and B. A. Robson, Int. J. Mod. Phys. E 15 (2006) 617. 
10. B. A. Robson, Int. J. Mod. Phys. E 14 (2005) 1151; B. A. Robson, Int. J. Mod. Phys. E 15 (2006) 273.

11. N. Cabibbo, Phys. Rev. Lett. 10 (1963) 531.

12. D. B. Leinweber et al., Phys. Rev. Lett. 97 (2006) 022001.

13. D. J. Gross and F. Wilczek, Phys. Rev. Lett. 30 (1973) 1343.

14. H. D. Politzer, Phys. Rev. Lett. 30 (1973) 1346.

15. F. Halzen and A. D. Martin, Quarks and Leptons: An Introductory Course in Modern Particle Physics (John Wiley and Sons, New York, 1984).

16. A. D. Morrison and B. A. Robson, Int. J. Mod. Phys. E 18 (2009) 1825.

17. S. G. Turyshev and V. T. Toth, Space Sci. Rev. 148 (2009) 149.

18. Y. Sofue and V. Rubin, Annu. Rev. Astron. Astrophys. 39 (2001) 137. 